\title{
The long-term optical behavior of Mkn 421
}

\author{
F.K. $\mathbf{L i u}^{1,2}$, B.F. $\mathbf{L i u}^{\star, 2}$, and G.Z. $\mathbf{X i e}^{2,3}$ \\ 1 International School for Advanced Studies, Via Beirut 2-4, 34013 Trieste, Italy \\ e-mail: fkliu@sissa.it \\ 2 Yunnan Observatory, Academia Sinica, P.O.Box 110, Kunming 650011, China \\ ${ }^{3}$ Center for Astrophysics, CCAST (World Laboratory), Beijing, China
}

Received February 6; accepted October 22, 1996

\begin{abstract}
All data available in $B$ band for the BL Lac object Mkn 421 from 22 publications are used to construct a historical light curve, dating back to 1900. It is found that the light curve is very complicated and consists of a set of outbursts with very large duration. The brightness of Mkn 421 varies from 11.6 magnitude to more than 16 magnitude. Analyses with Jurkevich method of computing periods of cyclic phenomena reveal in the light curve two kinds of behaviors. The first one is non-periodic with rapid, violent variations in intensity on time scales of hours to days. The second one is periodic with a possible period of $23.1 \pm 1.1$ years. Another possible period of $15.3 \pm 0.7$ years is not very significant. We have tested the robustness of the Jurkevich method. The period of about one year found in the light curves of Mkn 421 and of other objects is a spurious period due to the method and the observing window. We try to explain the period of $23.1 \pm 1.1$ years under the thermal instability of a slim accretion disk around a massive black hole of mass $210^{6} M_{\odot}$.
\end{abstract}

Key words: accretion, accretion disk - BL Lac objects: individual: Mkn 421

\section{Introduction}

Accretion disks play a fundamental role in the theoretical models of active galactic nuclei (AGNs). Investigations indicate that instability and pulsations in the inner, transonic regions with parameters in proper ranges have various time scales (for a review see Wallinder et al. 1992). The time scale of the dwarf nova type limit cycle instability in AGNs is too long to be observed directly for a central black hole of mass $10^{6} M_{\odot}$ or higher (Meyer-Hofmeister

Send offprint requests to: B.F. Liu

* Present address: Max-Plank-Institut für Astrophysik, KarlSchwarzschild-Str. 1, D-85740 Garching, Germany; e-mail: lbf@mpa-garching.mpg.de
1993), but the global thermal limit cycle (oscillation) time scale in slim accretion disks with $\alpha \sim 0.1$ around a central mass of $10^{6} M_{\odot}$ to $10^{10} M_{\odot}$ varies from a few years to several hundred thousand years (Honma et al. 1991). The latter periodic instability may be observed in some QSOs and BL Lac objects with available observational data for around 100 years.

Rapid and large amplitude variability for BL Lac objects, a special subclass of AGNs, has fostered considerable interest. The intra-day variability has been extensively investigated (see, e.g. Wagner \& Witzel 1995 and references therein). Only a few investigations, however, deal with the long-term variations of BL Lac objects due to the lack of data available over a long enough time scale. Therefore, whether periodic or quasi-periodic fluctuations on long term time scales exist is unclear. Several years ago, we started collecting observational data on some BL Lac objects and investigated their long time-scale variability. Liu et al. (1995) showed that ON 231 is a very active object with a probable periodic activity of $13.6 \pm 1.3$ years. This was interpreted as a thermal limit cycle (oscillation) in a slim accretion disk (for an alternative interpretation to the periodicity of OJ 87, see Sillanpää et al. 1988a). In this paper, we show that the BL Lac object Mkn 421 is also very active and probably has periodic activities.

The X-ray selected BL Lac object Mkn 421, at $z=$ 0.0308 has attracted much attention after it was identified as a BL Lac object. It is one of the objects simultaneously observed at all electro-magnetic frequencies and one of the few objects radiating strong gamma-rays. Many observations to search for its variability in optical waveband have been performed. Data from the archive plate collection of Harvard College Observatory showed a large range of variations, $\Delta B \geq 4.7$ magnitude (Miller 1975). After examining the data between 1974 and 1982, GagenTorn et al. (1983) concluded that Mkn 421 varied in $B$ band with an amplitude of 1.5 magnitude on characteristic time scales of days to years. The purpose of the present paper is to probe the long time-scale variability of $\mathrm{Mkn}$ 421. We collect all available observational data in $B$ band 
and give a general discussion on the light curves in Sect. 2 . In Sect. 3 we present a detailed analysis of the light curve, through the Jurkevich $V_{\mathrm{m}}{ }^{2}$ test. In order to test the robustness of the Jurkevich method, we analyze a simulated data set in Sect. 4. Final conclusions are given in Sect. 5 .

\section{Variability analysis of long-term light curves}

All observational data in $B$ band on Mkn 421 are available in the following studies: Miller (1975), Ulrich et al. (1975), Veron \& Veron (1975), Veron \& Veron (1976), Miller et al. (1977), O'Dell et al. (1978), Mufson et al. (1980), Puschell \& Stein (1980), Zekl et al. (1981), GagenTorn et al. (1983), Sitko et al. (1983), Cruz-Gonzalez \& Huchra (1984), Moles et al. (1985), Sitko et al. (1985), Makino et al. (1987), Xie et al. (1987), Sillanpää et al. (1988), Xie et al. (1988), Sillanpää et al. (1991), Sitko \& Sitko (1991), Takalo (1991), and Takalo et al. (1992). Data for Mkn 421 consist of 565 observations, dating back to 1900. Since we are searching for the long time variability, we include those data estimated from Miller's figures with relatively large date uncertainties (less than one month). The $B$-band observations are used in this paper because there are more data available in $B$-band than in other bands. We translate the photographic magnitude $m_{\mathrm{pg}}$ by the approximate relation $m_{B}=m_{\mathrm{pg}}+0.11$ and the flux density, $f_{B}$, by $m_{B}=\left[\log 4.4910^{6}-\log f_{B}(\mathrm{mJy})\right] / 0.4$ (Sitko et al. 1985).

The error caused by the conversion from photographic to photo-electric values is not larger than 0.2 magnitude. The object does not produce a stellar image in deep photographic exposures, so photometric data obtained with different entrance sizes are different. About half of our data are taken from Miller's paper (Miller 1975), where the uncertainty of the iris photometry measures are typically $0.1-0.2$ magnitudes. A large fraction of the remaining data are obtained with a diaphragm of about $26^{\prime \prime}$, within which the contribution of the host galaxy is less than 0.2 magnitude. Thus the difference between magnitudes derived through different entrance diaphragm sizes is less than about 0.2 magnitude. Therefore, the photometric and photo-electric data are consistent within 0.2 magnitude, a very small value compared to the large range of variation of the object, $\Delta B \geq 4.7 \mathrm{mag}$. The magnitude uncertainties introduce noise and introduce an uncertainty on the parameters of the temporal features possibly detected on the Jurkevich plot (see next section).

The long-term light curve is shown in Fig. 1a. Because of our purpose to investigate large-amplitude variations, we do not show individual error-bars. The effect of errors on the periodicity analysis will be discussed in Sect. 3. Mkn 421 is very active, with a range of variation of $\Delta B \geq 4.7$ mag. The source reached a maximal brightness of $11.6 \mathrm{mag}$ in 1934 January and was brighter than 12.5 mag on three occasions in 1901, 1916 and 1936 (Miller 1975). After reaching a maximum $B=12.75$ in 1982 April,
Mkn 421 faded out until 1986. There are fewer observations available for Mkn 421 after 1986, in $B$ band. The observations, however, still show that the source brightened again (Takolo 1991; Takolo et al. 1992).

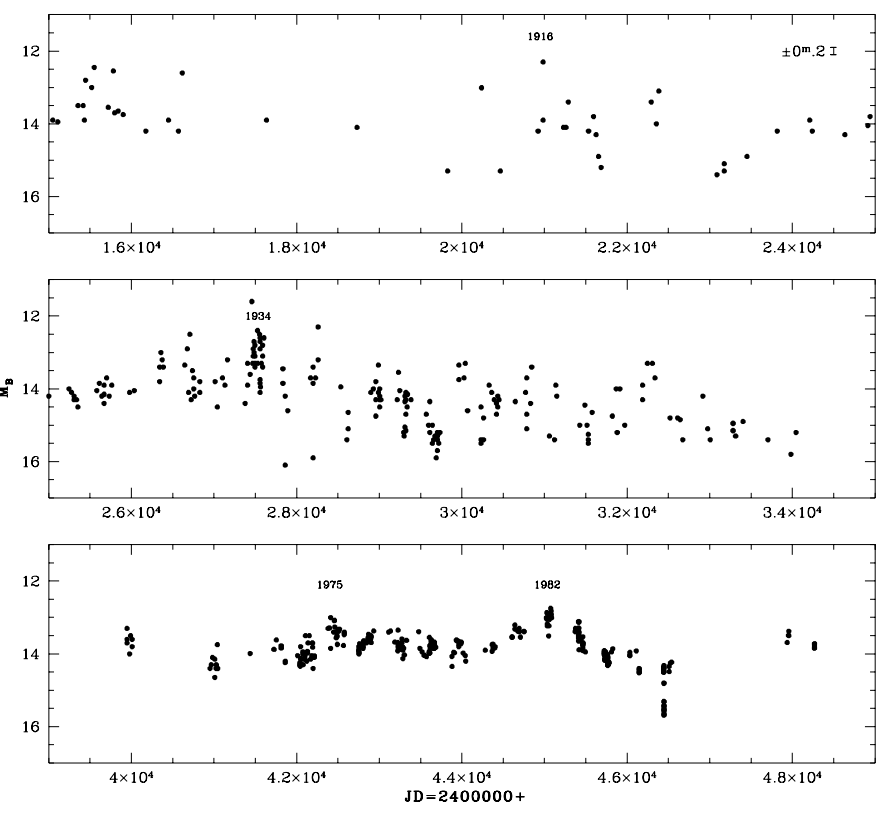

Fig. 1. a The long-term light curve of Mkn 421 from 1900 to 1991. The discontinuity of the light curve between 2435000 and 2439000 is due to lack of observations
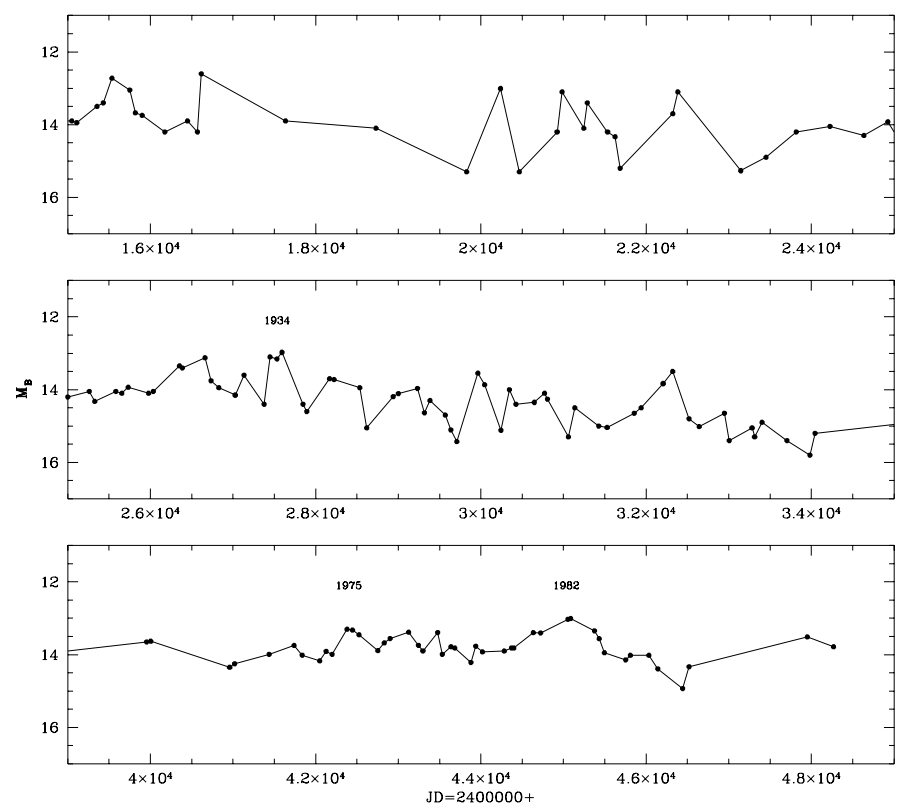

Fig. 1. b The mean light curve of Mkn 421 over a 100-day mean 
To reduce small amplitude intra-day's fluctuations, we averaged the light curve over 1 day. No significant difference has been found. In order to probe the longterm behavior of the variations, we averaged the light curve over 100-day (Fig. 1b). Because of the different quality of our data at different epochs, the impact of flickering in recent data is washed out,while still significant in the early data. As the object varies in intensity by about $0.5 \mathrm{mag}$ on a time scale of several hours (Xie et al. 1988), the largest difference between early epoch data point and the mean value can be estimated only within an uncertainty

of 0.5 mag. We have averaged the light curve. The resulting light curves are similar. The peaks in 1934, 1975 and 1982 remain unchanged. The difference between Figs. 1a and $b$ is quite significant. It indicates that Mkn 421 suffers large intensity variations on a time scale of a few months. The variability of Mkn 421 shows two modes: a short one with a time-scale of a few months to several years and a longer one with a time scale of the order of ten years. We will analyze the repetition of the bursts in the light curve using the Jurkevich method (1971) in Sect. 3.

\section{Periodic analysis}

Is there any period in the light curve? In this section, we try to answer this question using the powerful Jurkevich $V_{\mathrm{m}}^{2}$ method (Jurkevich 1971).

The Jurkevich method is based on the expected mean square deviation. It tests a run of trial periods around which the data are folded. All data are assigned to $m$ groups according to their phases around each trial period. The variance $V_{\mathrm{i}}^{2}$ of each group and the sum $V_{\mathrm{m}}^{2}$ of all groups are computed. For a trial period equal to the true one, if any, $V_{\mathrm{m}}^{2}$ reaches its minimum, and a "good" period will give a much reduced variance relative to those given by other false trial periods and with almost constant values. No firm rule exists for assessing the significance of a minimum in the $V_{\mathrm{m}}^{2}$ plot.

As in Kidger et al. (1992) and Liu et al. (1995), we consider the parameter $f$,

$f=\frac{\left(1-V_{\mathrm{m}}^{2}\right)}{V_{\mathrm{m}}^{2}}$

where $V_{\mathrm{m}}^{2}$ is the normalized value. In the normalized plot, a value of $V_{\mathrm{m}}^{2}=1.0$ implies that $f=0$ and hence there is no periodicity at all. The best periods can be identified from the plot. A value $f \geq 0.5$ generally indicates that a strong periodicity exists in the data, whilst $f<0.25$ usually indicates that the periodicity, if genuine, is a weak one. A further test is the relationship between the depth of the minimum and the noise in the "flat" section of the $V_{\mathrm{m}}^{2}$ curve close to the adopted period. If the absolute value of the relative change of the minimum to the "flat" section is larger than ten times the standard error of this "flat" section, the periodicity in the data can be considered as significant and the minimum as highly reliable. In the Jurkevich test the parameter $m$ can be modified: more groups give higher sensitivity, but fewer data points per group introduce a larger noise in the plot. So we analyze the data sample mainly using $m=10$, which gives us over 50 points per group. To search for short time scale periods, we choose a small interval between two successive trial periods.

The result of the analysis with $m=10$ is shown in Fig. 2. A minimum of $V_{\mathrm{m}}^{2}=0.653(f=0.532)$ is significant at a trial period of $23.1 \pm 1.1$ years. A similar analysis with $m=20$ shows that $V_{\mathrm{m}}^{2}=0.615(f=0.627)$ at the period of 23.5-year. In addition to the period of $23.1 \pm 1.1$ years, the broad minimum at $P=15.5$ years is also significant with $V_{\mathrm{m}}^{2}=0.701$ and $f=0.427$ but not as certain as the one obtained at $P=23.1$ years. We have considered the half width at half minimum as the "formal" error (cf. Jurkevich 1971) to derive all effects on the precision, including random variations in the exact interval between outbursts, poor coverage of some of the early outbursts and the larger error in some of the early photographic photometry, the uncertainty of observed data estimated from the figures in the literature, random variations in intensity, and the changing width of the outburst structure. The errors caused by the conversion from photographic to photo-electric values and by the measurement with different diaphragms are considered in this analysis as random variations in the intensity. They would reduce the depth of minima and therefore the significance of the periodicity found. These errors also increase the "formal" error, and this effect has been taken into account. The fluctuations seen around the minimum may also be caused by flickering, which is definitely non-periodic. The broad width of the minima may also result from the broad structures of the bursts, the drift of the real period and the effect of adjacent periods, if present.

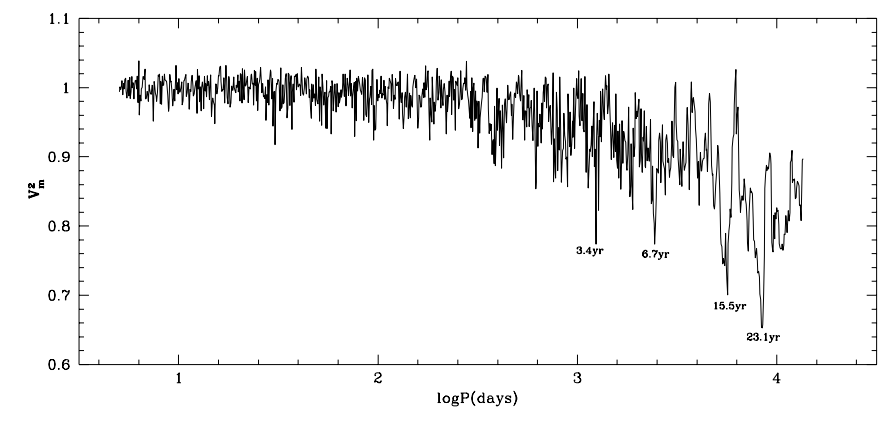

Fig. 2. Results of the normalized Jurkevich test for the period search, in Mkn 421. The deepest minimum corresponds to a period of 23.1 years. The minima corresponding to periods of 3.4 years, 6.7 years, and 15.5 years are also conspicuous 


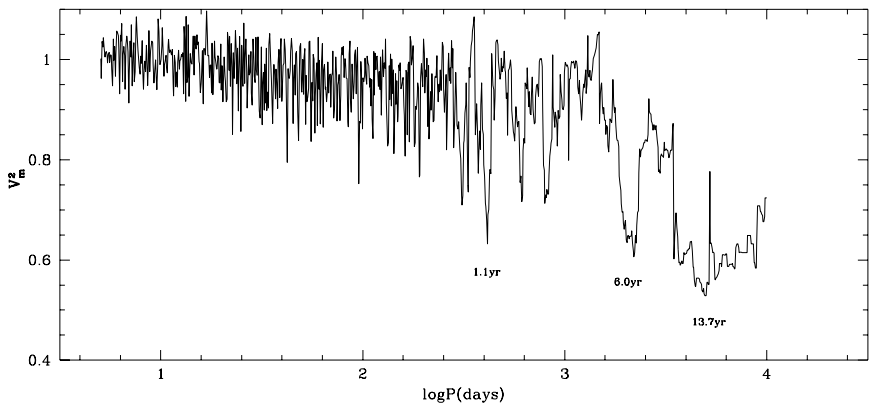

Fig. 3. Results of the normalized Jurkevich test for the period search, in Mkn 421, excluding Miller's data, with $m=5$. The minima corresponding to periods of $1.1,6.0$, and 13.7 years are significant

To compensate for the heavy weighting of recent data, we use the 100-day averaged light curve. This interval is long enough compared to the possible periods of 15.3 years and 22.8 years and unlikely to prevent a distribution of the long term variation findings. The result of the Jurkevich test shows a larger noise due to fewer points in every group and flickering effects in the early epochs. However, a minimum of $V_{\mathrm{m}}^{2}$ near the possible period of 22.8 years is still seen.

Considering the redshift of 0.0308 , the period of 23.1 years corresponds to 22.4 years in the rest frame of the source.

\section{The robustness of the Jurkevich method}

In order to test the robustness of the Jurkevich method and to investigate intermediate time-scale periods, we exclude Miller's data and use the observational data from 1972 to 1991. During this period, Mkn 421 was more extensively monitored and thus has sufficient data for our analysis to give reliable results. The result of the analysis, with $m=5$, is shown in Fig. 3 .

Although the time interval considered in this case is less than that covered in Fig. 2 (only 19 years), a minimum at $P=13.7 \pm 2.0$ years is quite significant and broad, and consistent with the results given in Fig. 2. This confirms the period of 15.3 years and test the robustness of the Jurkevich method.

In addition to the minimum at 13.7 years, a second minimum at period $P=6.0$ years with $V_{\mathrm{m}}^{2}=0.606$ and $f=0.649$ is found to be significant and broad. Its relative depth, however, is only about 8 times the nearby noise. Although the period of $P=6.0$ years is about half the period of $P=13.7$ years, we cannot be sure of the reality of the former, as it does not appear in Fig. 2.

In addition to the possible periods of $22.8,15.3$ and 6.0 years, the plots also show minima at $P=1.1,2.2$ and 3.4 years in Figs. 2 and 3 with relatively less significance. A one-year period was also found in the light curves of ON 231 (Liu et al. 1995) and 3C 120 (Jurkevich et al.
1971). A one year period is doubtful as the astronomical cycle is of one year. In order to check whether the period is a spurious result of the Jurkevich method, we did the following test.

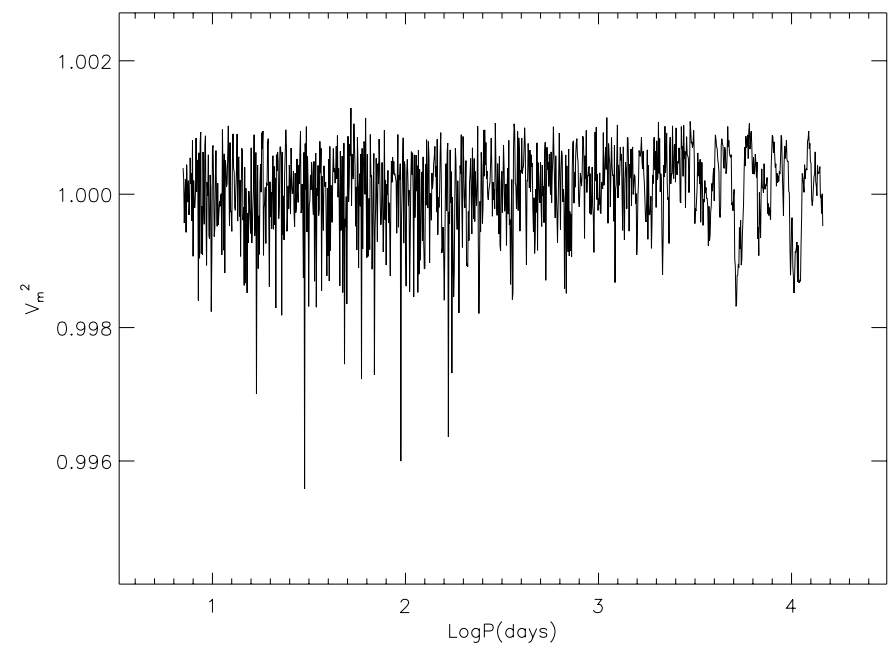

Fig. 4. The plot of normalized $V_{\mathrm{m}}^{2}$ vs. trial period for a test object with random variations. No significant minima corresponding to trial periods of one year and multiple are found in the plot

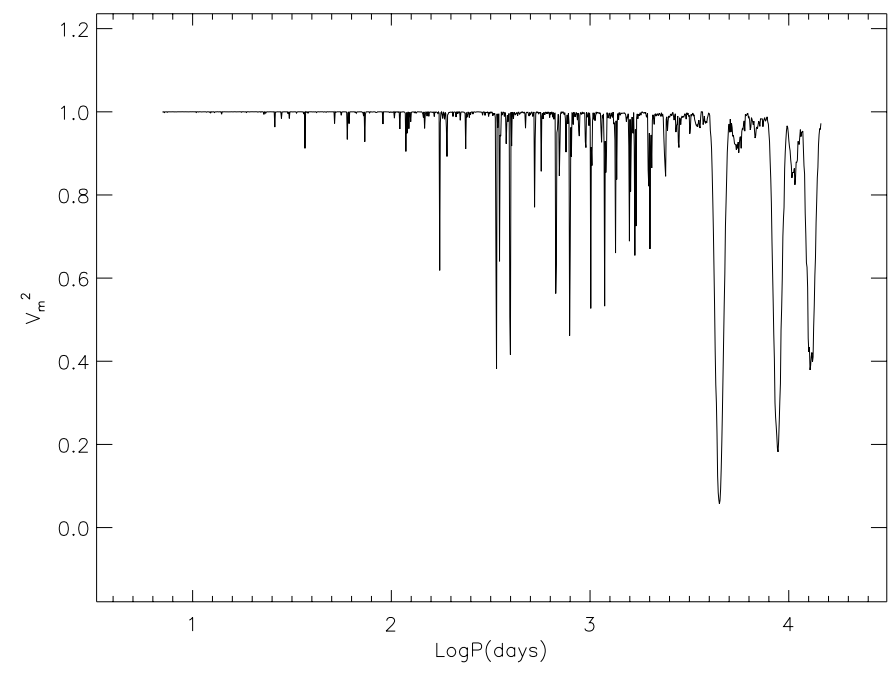

Fig. 5. Same as Fig. 4 for a test object with sinusoidal variation of a period of 12.5 years. In addition to the minima at $P=12.5$ years and multiple, the minima corresponding to a period of one year and multiple are significant and are artifacts of the method used

To test the method, we take an object with only random variations with an amplitude of 4 magnitudes. To mimic real observations, we make a further assumption that the object can been observed only from the begin- 
ning of January to the end of March every year and that the available data covers a one hundred year range. We also assume that, for moon light reasons for example, it can been observed only for 10, 20 or 30 days a month. Under these assumptions, the number of data points (one point a day) would be 3000, 6000 and 9000 . The result of the Jurkevich analysis for the 20-day case is shown in Fig. 4. The results for the others are similar. No significant minima are found at one year and multiple. When we change the assumption from three months to four months and do the test again, the conclusion is unchanged. Now, we assume that the source varies sinusoidally with a period of 12.5 years and we keep all the other assumptions. The result of the analysis for the 20-day case is shown in Fig. 5. In addition to the minima at 12.5 years and multiple, the minima at one year and multiple become very significant. If we assume we could observe 12 months a year, the minimum corresponding to a period of one year does not exist any more in the $V_{\mathrm{m}}^{2}$ plot. We conclude that the Jurkevich method does not give a spurious period of one year for a randomly variable source, but if there exists a long term period in the light curve of the source, a spurious period of around one year will appear. This can probably be understood as follows: when the trial period is slightly different from half a year, and one year and its multiple, some of the ten groups $(m)$ contain very few observational points which lead to a very small variance $V_{\mathrm{i}}^{2}$ and therefore small $V_{\mathrm{m}}^{2}$ (cf. Fig. 5). So the minima at about one year and multiple might be taken as another signal of the existence of a long time-scale period in the light curve.

The Jurkevich analyses of the observational data with Miller's and without Miller's data provide similar results. These results are independent of the parameter $m$. Our analysis shows that probably two periods exist in the light curve of the BL Lac object Mkn 421: one of around 15 years and another of around 23 years. However, the current data set covers only four times the possible period of 23 years, so more data is needed to confirm this 23 years period.

\section{Conclusions}

We assembled the historical light curve of the BL Lac object Mkn 421 and searched for its possible periodicity using the Jurkevich method. Our results indicate that this object is very active and probably has two periodic activities. One period is of $23.1 \pm 1.1$ years and the other is of $15.3 \pm 0.7$ years which, if real, superposes on the former. The former period has a higher confidence. The period of 23 years is about half the time interval between the well observed outbursts in 1934 and 1982. If the period is real, outbursts probably occurred between 1953 and 1968, where unfortunately, no published data are available. We must remember, however, that there is some noise on the curve (Fig. 2) and that the total observation range spans only four times the period of 23 years. More observations are required to assess the reality of this period.

The period of one year and multiple found in Mkn 421, in ON 231 (Liu et al. 1995) and in 3C 120 (Jurkevich et al. 1971) are spurious results due to the existence of a long time-scale period and a cycle of one year in the astronomical optical observations.

Regarding the 23 years period, we tentatively provide below a theoretical explanation. Sillanpää et al. (1988a) suggest a binary black hole model to explain the quasi-periodic behaviour found in BL Lac object OJ 287. However, there are several difficulties with the binary model: observed periodicity is not exact, the period in OJ 287 corresponding to the minimum of brightness is quite doubtful, observed burst structures are very broad, the system is short-lived due to gravitational radiation and dynamically unstable due to the interaction between secondary black hole and disk. Periodicity has probably been found in many BL Lac objects (Liu 1996) and therefore is not probably at the origin of binary black holes. The fact that the duration of a burst is around half the quasi-period can be interpreted in terms of thermal instabilities in a slim accretion disk in AGNs. Some simulations have shown that slim disks can indeed be subject to limit-cycle type oscillations, as in the case of dwarf novae although with a different oscillation behavior (Taam \& Lin 1984; Lasota \& Pelat 1991; Honma et al. 1991). The basic characteristics of the thermal limit cycles depend strongly on the viscosity parameter $\alpha$, central black hole mass $M_{6}=\frac{M}{10^{6} M_{\odot}}$, accretion rate $\dot{M}$ and generalized stress tensor parameter $\mu$ (cf. Wallinder et al. 1992). However, the time duration of the bursts is almost independent of both $\mu$ and $\dot{M}$, and may be written empirically as

$t_{\text {burst }} \simeq 4.5 \alpha_{0.1}^{-0.62} M_{6}^{1.37}$ yrs,

when $\mu=0.5$ and $\dot{M} \simeq 0.2 \dot{M}_{\mathrm{c}}$ where $\dot{M}_{\mathrm{c}}=\dot{M}_{\mathrm{E}} / \epsilon, \dot{M}_{\mathrm{E}}$ being the Eddington accretion rate and $\epsilon$ the accretion efficiency (Honma et al. 1991). The time interval between subsequent bursts depends strongly on $\mu$, but weakly on $\dot{M}$. As both the origin and the properties of the presumed viscosity in accretion disks are unknown at present, its hydro-magnetic origin is one of the options. (Horiuchi \& Kato (1990) suggested that $\mu \simeq 0.5$ may hold if the escape rate of the magnetic field is low. With these values of the parameters, the thermal limit cycle time $t_{\mathrm{cyc}}$ (period) should be of the order of $2 t_{\text {burst }}$, i.e.

$t_{\mathrm{cyc}} \sim 9.0 \alpha_{0.1}^{-0.62} M_{6}^{1.37} \quad \mathrm{yrs}$.

For Mkn 421, if we adopt the typical values of $\alpha=0.1$, $\mu=0.5$ and $\dot{M} \simeq 0.2 \dot{M}_{\mathrm{c}}$ and search the central black hole mass $M$ to get a period of 22.4 years, we find an estimated mass of $M \simeq 210^{6} M_{\odot}$. This mass is reasonable if the parent galaxies of BL Lac objects are FR I radio galaxies. 
Acknowledgements. We are grateful to the referee, Dr. D Alloin, for her helpful comments.

\section{References}

Cruz-Gonzalez I., Huchra J.P., 1984, AJ 89, 441

Gagen-Torn V.A., Marchenko S.G., Smekhacheva R.I., Yakovleva V.A., 1983, Astrophys. 19, 111

Honma F., Matsumoto R., Kato S., 1991, PASJ 43, 147

Horiuchi T., Kato S., 1990, PASJ 42, 661

Jurkevich I., 1971, Ap\&SS 13, 154

Kidger M.R., Takalo L., Sillanpaa A., 1992, A\&A 264, 32

Lasota J.P., Pelat D., 1991, A\&A 249, 574

Liu F.K., 1996, in Proc. 21st Cent. Chin. Astron. Conf., held in Hong Kong on 1-4 August, Cheng K.S. et al. (eds.) (in press)

Liu F.K, Xie G.Z., Bai J.M., 1995, A\&A 295, 1

Makino F., Tanako Y., Matsuoka M., et al., 1987, ApJ 313, 662

Meyer-Hofmeister E., 1993, in Proceedings of 4th MPG-CAS Workshop on High Energy Astrophysics and Cosmology, Börner G., Buchert T. (eds.), MPA/P8, p. 126

Miller H.R., 1975, ApJ 201, L109

Miller H.R., McGimsey B.Q., Williamon R.M., 1977, ApJ 217, 382

Mufson S.L., Wisniewski W.Z., Wood K., et al., 1980, ApJ 241, 74
Moles M., Garcia-Pelayo J., Masegosa J., Aparicio A., 1985, ApJS 58, 255

O'Dell S.L., Puschell J.J., Stein W.A., Warner J.W., 1978, ApJS 38, 267

Puschell J.J., Stein W.A., 1980, ApJ 237, 331

Sillanpää A., Haarala S., Korhonen T., 1988, A\&AS 72, 347

Sillanpää A., Haarala S., Valtonen M., Sundelius B., Byrd G.G., 1988a, ApJ 325, 628

Sillanpää A., Mikkola S., Valtaoja, L., 1991, A\&AS 88, 225

Sitko M.L., Schmidt G.D., Stein W.A., 1985, ApJS 59, 323

Sitko M.L., Stein W.A., Zhang Y.-X., Wisniewski W.Z., 1983, PASP 95, 724

Sitko M.L., Sitko A.K., 1991, PASP 103, 160

Taam R.E., Lin D.N.C., 1984, ApJ 287, 761

Takalo L.O., 1991, A\&A 90, 161

Takalo L.O., Sillanpää A., Nilsson K., et al., 1992, A\&AS 94, 37

Ulrich M.-H., Kinman T.D., Lynds C.R., Rieke G.H., 1975, ApJ 198, 261

Veron P., Veron, M.P., 1975, A\&A 39, 281

Veron P., Veron M.P., 1976, A\&AS 25, 287

Wallinder F.H., Kato S, Abramowicz M.A., 1992, A\&AR 4, 79

Wagner S.J., Witzel A., 1995, ARA\&A 33, 163

Xie G.Z., Li K.H., Bao M.X., et al., 1987, A\&AS 67, 17

Xie G.Z., LU R.W., Zhou Y., et al., 1988, A\&AS 72, 163

Zekl H., Klare G., Appenzeller I., 1981, A\&A 103, 342 